\title{
Editorial
}

\section{The Urgency of Expanding Pre-Exposure Prophylaxis (PrEP) for Global HIV Reduction}

\author{
Douglas A. Feldman, PhD \\ Professor Emeritus, Department of Anthropology, SUNY Brockport, 350 New Campus Drive, Brockport, NY I4420, USA

\section{"Corresponding author} \\ Douglas A. Feldman, PhD \\ Professor Emeritus, Department of Anthropology, SUNY Brockport, 350 New Campus Drive, Brockport, NY I4420, USA; \\ E-mail: dfeldman@brockport.edu
}

\section{Article information}

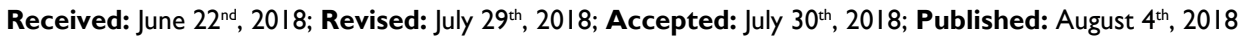

\section{Cite this article}

Feldman DA. The urgency of expanding pre-exposure prophylaxis (PrEP) for global HIV reduction. Anthropol Open J. 20I8; 3(I): el-e3. doi: I0.17|40/ANTPOJ-3-e004

G lobally, an estimated 76.1 million persons have become in fected with HIV since the start of the epidemic, with 35 million having died from AIDS-related illnesses. In spite of 19.1 billion dollars spent annually in low- and middle-income countries, with most of those funds going toward the provision of antiretroviral medications, only 56.9 percent of all HIV-infected persons are receiving these medications. While the number of new HIV infections have been declining recently, 1.8 million people were newly infected in 2016 .

Back in the 1980's and 90's, some of us medical anthropologists, HIV prevention researchers and interventionists wished that there would someday be a pill that one could take that would work as well as, or better than, a condom in preventing HIV/AIDS. ${ }^{2}$ In fact, that day has actually come. Today, and since 2012, there is such a pill, and most persons globally, including many health professionals, still do not know about it. While newer versions are currently in development, the most commercially available version is Truvada, generically referred to as pre-exposure prophylaxis (PrEP), which is a combination antiretroviral drug (tenofovir/ emtricitabine). PrEP has been shown to be highly effective in preventing HIV in both research studies and reports from the field, greater than 90 percent, especially if taken as directed. ${ }^{3}$

The current recommendation is for people at high risk for HIV/AIDS to take the pill once daily and to be tested for HIV every three months for adults, and every month for teenagers. ${ }^{4}$ High-risk groups include men who have sex with men (MSM), transwomen, female sex workers (FSW), male sex workers, injecting drug users (IDU), serodiscordant couples (where one partner is HIV positive and the other HIV negative), and young sexually ac- tive women. ${ }^{5-10}$ Certainly, not everyone in these "high risk groups" are truly high risk and do not need to take PrEP.

MSM refers to same-sex sexual behavior, and include gay-identified, bisexual-identified, and straight-identified men who engage in sex with other men. MSM, for example, who use condoms every time, or are in a mutually exclusive relationship based on trust and fidelity, or do not have anal sex, or do not have sex at all, are not at high risk for HIV infection. IDUs who never share needles with others and routinely engage in safer sex are also not at high risk. However, for most sexually active men and women who do not consistently use condoms, PrEP has been shown to be safe and effective in reducing the risk for HIV, even more effectively than condoms themselves. ${ }^{11,12}$ PrEP used with condoms is estimated to be 99 to 100 percent effective in preventing HIV, even with a known HIV positive partner.

The medication originally was used in the treatment of HIV as an antiretroviral drug, belonging to a class of HIV nucleoside reverse transcriptase inhibitor drugs. There are very few potential side effects to PrEP when used in healthy HIV negative persons. Lymphadenopathy, for example, which does sometimes occur when used as a treatment for HIV positive persons, has not been observed among PrEP users. However, individuals with kidney disease or osteoporosis should not use PrEP, since it could worsen these two conditions.

The majority (69.5 percent) of persons with HIV infection are located in sub-Saharan Africa. Of the 36.7 million persons worldwide living with HIV in 2016, 19.4 million were in eastern and southern Africa, and 6.1 million were in western and 
central Africa. ${ }^{1}$ The President's Emergency Plan For AIDS Relief (PEPFAR) is the US-funded program for HIV prevention, HIV treatment, and AIDS care in Africa and elsewhere, and the largest AIDS-related international donor organization in the world. Other major donors are the mostly European-sponsored Global Fund to Fight AIDS, Tuberculosis, and Malaria; and the Bill and Melinda Gates Foundation. ${ }^{13}$

PrEP has been available for six years, and to date only 200,000 persons worldwide are using this crucial medication. Most of that number, are in the United States: 150,000 Americans mostly MSM are using PrEP, while a mere 50,000 throughout the rest of the world today are using the drug. ${ }^{14}$ The scale-up of PrEP use in Africa and elsewhere has been unacceptably slow. In many countries, it is simply unavailable. ${ }^{15}$ By now, tens of millions of HIV negative men and women who are at high risk for HIV infection, or are simply living in countries where the adult HIV seroprevalence remains in the double digits, should be taking PrEP on a daily basis to prevent HIV/AIDS. In Africa, Kenya - with 13,000 PrEP users, appears to be the most capable nation in beginning to promote the uptake of the drug, but they still have a long way to go to be successful. ${ }^{14}$

Impediments to proliferating the use of PrEP in Africa include: 1) intense stigma against MSM, FSW, and IDUs, which makes it difficult to identify and reach out to these high-risk persons; ${ }^{16,17}$ 2) the expense of the medication; 3) the need to conduct quarterly HIV tests to ensure that PrEP is not given to users who should become HIV positive; 4) the need to conduct periodic creatinine tests, since it may adversely affect the kidneys in some users, and 5) the dilemma in which many teenage and young adult females often do not see themselves at risk for HIV even though they are sexually active, their partners do not use condoms, and they live in a country where AIDS is endemic. ${ }^{18,19}$

PEPFAR recognizes the need to rapidly scale-up the availability and acceptance of PrEP in Africa and elsewhere. . $^{13,20,21}$ However, in its recent guidelines on how to achieve that goal, it argues that little additional funds need to be allocated specifically for PrEP, and that most of the scale-up can be done with the existing structure and funds of their prevention and treatment program. However, it is clear that getting millions of Africans and others to use PrEP on a daily basis will be costly and require new allocated funds. These need to be designated specifically for the purchase of the medication, expansion of HIV and creatinine testing, prevention programs to get the word out to both the general public and high risk groups, evaluation research by medical anthropologists and other social/behavioral scientists to ensure the success of the programs, and programs to destigmatize negative attitudes facing high risk groups. In short, PEPFAR and other donor organizations need to take PrEP seriously, if there is any hope to significantly lessen the impact of HIV/AIDS by their stated 2030 goal for the eradication of AIDS worldwide.

The time has come to rapidly scale-up the proliferation of PrEP throughout Africa and worldwide if there is to be any hope of ending this global pandemic. As Mugo and colleagues stated,
"Failure to implement PrEP will be a failure to protect future generations". ${ }^{22}$ Medical anthropologists have played a substantial role in understanding the AIDS crisis since as early as $1982 .{ }^{23-24}$ We can, and should, play an important and decisive role in achieving this goal.

\section{ACKNOWLEDGEMENT}

I would like to thank Dr. William Valenti, Ashley Zuppelli, and the staff of Trillium Health - the AIDS service center of Rochester, NY for introducing me to their excellent PrEP program in preparation for this editorial.

Dr. Feldman is a Professor Emeritus and former Department Chair of Anthropology at The College at Brockport, State University of New York, and is the former President of the Society for Medical Anthropology. He is the author/editor of seven books, and has conducted AIDS social and epidemiologic research in Hungary, Rwanda, Senegal, Uganda, the United States, and Zambia.

\section{REFERENCES}

1. UNAIDS. Fact sheet World AIDS Day 2017. Communications and Global Advocacy.

2. CDC. Pre-exposure prophylaxis (PrEP) for HIV prevention. May 2014.

3. AVAC: Global Advocacy for HIV Prevention. Pre-exposure prophylaxis (PrEP) - An introductory factsheet. November 2017.

4. Treatment News. Gay and bi teens may need monthly monitoring to adhere well to PrEP. 2016.

5. Bazzi AR, Yotebieng KA, Agot K, Rota G, Syvertsen JL. Perspectives on biomedical HIV prevention options among women who inject drugs in Kenya. AIDS Care. 2018; 30(3): 343-346. doi: 10.1080/09540121.2017.1363369

6. Cremin I, McKinnon L, Kimani J, et al. PrEP for key populations in combination HIV prevention in Nairobi: A mathematical modeling study. Lancet HIV. 2017; 4(5): e214-e222. doi: 10.1016/ S2352-3018(17)30021-8

7. Eakle R, Bourne A, Mbogua J, Mutanha N, Rees H. Exploring acceptability of oral PrEP prior to implementation among female sex workers in South Africa. J Int AIDS Soc. 2018; 21(2): e25081. doi: 10.1002/jia2.25081

8. Koss CA, Ayieko J, Mwangwa F, et al. Early adopters of HIV pre-exposure prophylaxis in a population-based combination prevention study in rural Kenya and Uganda. Clin Infect Dis. 2018. doi: $10.1093 / \mathrm{cid} / \operatorname{ciy} 390$

9. Pyra M, Haberer JE, Heffron R, et al. Partners Demonstration 
Project Team. Brief report: PrEP use during periods of HIV risk among East African Women in serodiscordant relationships. J Acquir Immune Defic Syndr. 2018; 77(1): 41-45. doi: 10.1097/ QAI.0000000000001561

10. Sithole B. HIV prevention needs for men who have sex with men in Swaziland. Afr J AIDS Res. 2017; 16(4): 315-320. doi: $10.2989 / 16085906.2017 .1379420$

11. Hancuch K, Baeten J, Ngure K, et al. Safer conception among HIV-1 serodiscordant couples in East Africa: Understanding knowledge, attitudes, and experiences. AIDS Care. 2018. 17: 1-9. doi: 10.1080/09540121.2018.1437251

12. Heffron R, Ngure K, Odoyo J, et al. Pre-exposure prophylaxis for HIV-negative persons with partners living with HIV: Uptake, use, and effectiveness in an open-label demonstration project in East Africa. Gates Open Res. 2017; 1: 3. doi: 10.12688/gatesopenres.12752.1

13. PrEP Scientific Advisory Board. Recommendations on the use of PrEP for all populations. 2018; PrEP Expert Working Group, PEPFAR: USA. Department of State.

14. Cohen J. Concern as HIV prevention strategy languishes. Science. 2018, 359(6381): 1205. doi: 10.1126/science.359.6381.1205

15. UNAIDS. PrEP in South Africa. 2016. Feature story.

16. Rodriguez-Hart C, Nowak RG, Musci R, et al. Pathways from sexual stigma to incident HIV and sexually transmitted infections among Nigerian MSM. AIDS. 2017; 31(17): 2415-2420. doi: 10.1097/QAD.0000000000001637
17. Hartmann M, McConnell M, Bekker LG, et al. Motivated reasoning and HIV risk? Views on relationships, trust, and risk from young women in Cape Town, South Africa, and implications for oral PrEP. AIDS Behav. 2018; pp. 1-12. doi: 10.1007/s10461-0182044-2

18. Van der Wal R, Loutfi D. Pre-exposure prophylaxis for HIV prevention in East and southern Africa. Can J Public Health. 2018; 108(5-6): e643-e645. doi: 10.17269/cjph.108.6254

19. Nelson L. Pre-exposure prophylaxis: Perspectives from PEPFAR. 2018; PEPFAR: USA. Department of State. (Slide presentation).

20. PEPAR 2018 Country Operation Plan Guidance for Standard Process Countries. 2018; PEPFAR: USA. Department of State.

21. Mugo NR, Ngure K, kiragu M, Irungu E, Kilonzo N. PrEP for Africa: What we have learnt and what is needed to move to program implementation. Curr Opin HIV AIDS. 2016; 11(1): 80-86. doi: $10.1097 / \mathrm{COH} .0000000000000224$

22. Feldman DA. AIDS, culture, and Africa: The anthropological perspective. In: AIDS, Culture, and Africa (ed.: DA Feldman). Gainesville, USA: University Press of Florida. 2008. Pp. 1-17

23. Feldman DA. AIDS, culture, and gay men: An introduction. In: AIDS, Culture, and Gay Men (ed.: DA Feldman). Gainesville, USA: University Press of Florida. 2010. Pp. 1-20.

24. Whelehan P. The Anthropology of AIDS: A Global Perspective. Gainesville, USA: University Press of Florida. 2009. 